\title{
Novel rhodanine based molecular acceptor for organic solar cells
}

\author{
Surya Subianto ${ }^{1}$, Mats Andersson ${ }^{1, a}$, Naba Dutta ${ }^{1,2}$, and Namita Roy Choudhury ${ }^{1,2, *}$ \\ ${ }^{1}$ Future Industries Institute, University of South Australia, Adelaide 5095, Australia \\ 2 School of Chemical Engineering, University of Adelaide, Adelaide 5005, Australia
}

Received: 14 April 2017 / Received in final form: 30 July 2017 / Accepted: 29 August 2017

\begin{abstract}
A dirhodanine-substituted benzothiadiazole compound has been synthesised using Knoevenagel condensation of a dialdehyde-substituted benzothiadiazole and rhodanine. The resulting compound was deep orange red in colour and shows a HOMO and LUMO levels of -5.61 and $-3.85 \mathrm{eV}$ respectively, which makes it suitable for applications such as acceptor for organic solar cells.
\end{abstract}

Keywords: Organic solar cell / Molecular acceptor / 2,1,3-Benzothiadiazole / Rhodanine

\section{Introduction}

Amongst the current solar cells, organic, bulk heterojunction solar cells represent a highly promising alternative to conventional, silicon based solar cells due to their potential low-cost manufacturing and versatility derived from the plastics and printing industries. These devices have seen significant advances in recent years, with a large gain in efficiencies which are now approaching those of inorganic solar cells. One of the greatest leap in efficiency came with the introduction of solution-processable fullerene derivatives, whose introduction as organic n-type acceptor have led to improvement in the design and efficiencies of these organic solar cells. Indeed, their success means that, despite the high cost of fullerene materials, comparatively little has been reported on non-fullerene small molecule acceptors. However, fullerenes have their limitations, such as their wide bandgaps and low absorbance in the visible region, difficult functionalization to modify their energy level and absorption, air degradation issues and undesirable mechanical properties due to film brittleness as well as cost incurred due to their energyintensive synthesis [1]. Thus, development of novel alternative acceptors, which can overcome the drawbacks of fullerene but mimic the superior electron transporting behaviour of fullerene, is highly sought after.

Recently, non-fullerene acceptors [2-6], with n-type organic molecule instead of a fullerene derivative as the electron-acceptor material, have gained rapid significant

\footnotetext{
${ }^{a}$ Present address: Flinders Centre for Nanoscale Science and Technology, Flinders University, Sturt Road, Bedford Park, Adelaide, SA 5042, Australia.

* e-mail: namita.roychoudhury@adelaide.edu.au
}

interest in the field of organic solar cells (OSC). Replacing traditional fullerene acceptors in the photoactive layer of a normal organic solar cell with the organic acceptors offers several advantages, like light absorption and energy level tunability, diversity of donor-to-acceptor combination, and large-scale production of acceptor materials. However, the development of non-fullerene electron acceptor materials has fallen far behind that of large number of new donor materials. Recent work in this area has shown great promise in the use of molecules containing heterocycle units such as carbazole or benzothiadiazole linked through a conjugated backbone. Compounds such as vinazene [7], diketopolypyrrole [8], fluorine [9], and others were amongst those that have attracted research attention and have met varying degrees of success as molecular acceptors $[1,10]$. Besides, their synthetic reproducibility and diverse range of attainable structures make them very attractive for making materials with easy energy level control, mobility, solid packing and solubility. Certainly these small molecule acceptors also should satisfy the prerequisites: solar photon capturing ability, electron affinity and mobility, and self-assembling and phaseseparation ability related to the formation of nanoscale interpenetrating networks with the blended donor material. While significant progress has been made in the design and synthesis of these molecules; however the limiting factor is the active layer morphology, where, it may be difficult to form a well-ordered interpenetrating network due to the donor and acceptor being too miscible or over crystallisation of one or both components. A recent study indicated that an optimal morphology may lie in between the two states, with the necessity to have both a crystalline, pure domain of donors and acceptors within a matrix of an amorphous, miscible donor-acceptor $\operatorname{mix}[11]$. 
More recently, the introduction of dye end units into these types of compounds effectively improved light absorption and electron affinity, improving their performance as an electron acceptor. Rhodanine derivatives have been used as acceptor moieties in a variety of such push-pull compounds in fields such as nonlinear optics and dye-sensitised solar cells [12]. Studies of push-pull chromophores with dicyanomethylene rhodanines have shown significant electronic communication between the electron rich units and the rhodanine units [13]. In OPV, small molecule donor containing 3-ethylrhodanine dye has been recently developed that achieved a very high efficiency of up to $9 \%[14,15]$ and compounds with rhodanine end groups have shown good efficiencies of over $4 \%$ when used as acceptor in OPV (with poly-3hexylthiophene as donor) [16,17]. In this communication, we present the facile synthesis of a molecular acceptor based on 2,1,3-benzothiadiazole flanked with two rhodanine units. The compound was synthesised by Knoevenagel condensation between a disubstituted benzothiadiazole and rhodanine. Their optical and electrochemical properties were investigated, showing their potential for use as acceptors.

\section{Experimental}

\subsection{Materials}

2,1,3-benzothiadiazole, rhodanine, $48 \%$ hydrobromic acid $(\mathrm{HBr})$, acetic acid (glacial), N,N-dimethylformamide (DMF), sodium periodate $\left(\mathrm{NaIO}_{4}\right)$, trioxane, myristyltrimethylammonium bromide, and dimethyl sulfoxide were purchased from Aldrich and used as received. N,Ndimethylformamide (DMF) was purchased from Aldrich and dried by azeotropic distillation $(15 \mathrm{~mL}$ toluene in $250 \mathrm{~mL}$ DMF) prior to use.

\subsection{Instrumental analysis}

NMR analysis was done on a Bruker $300 \mathrm{MHz}$ NMR. UVVis spectroscopy was done using an Evolution $201 \mathrm{UV} / \mathrm{Vis}$ spectrophotometer. Samples were dip-coated onto glass slides from a solution in DMSO for measurements. Cyclic voltammetry was done using a Solartron 1260 Potentiostat in a three electrode setup with a glassy carbon working electrode, a platinum wire as counter electrode, and $\mathrm{Ag}$ / $\mathrm{AgCl}$ reference. $\mathrm{RhBT}$ was deposited onto a glassy carbon electrode for analysis. The CV was done in $0.1 \mathrm{M}$ solution of 1-butyl-3-methylimidazolium hexafluorophosphate in acetonitrile. The solution was degassed with argon for $1 \mathrm{~h}$ prior to analysis. The reference was calibrated against ferrocene redox couple.

\subsection{Synthesis of 4,7-di(bromomethyl)-benzo $(1,2,5)$ thiadiazole (1) (BBT)}

2,1,3-benzothiadiazole $(2 \mathrm{~g}, 14.6 \mathrm{mmol})$, trioxane $(4 \mathrm{~g}$, $20.8 \mathrm{mmol}$ ), and myristyltrimethylammonium bromide $(0.16 \mathrm{~g})$ were dissolved in $48 \% \mathrm{HBr}(80 \mathrm{~mL})$ and glacial acetic acid $(20 \mathrm{~mL})$ and the solution was stirred at $80^{\circ} \mathrm{C}$ for 3 days under nitrogen. The solution was then cooled and added to $100 \mathrm{~mL}$ of water, after which the solids were filtered and washed with deionised water and ethanol and the filtered solids were dried under vacuum and recrystallised from toluene to obtain compound 1. Yield: $2.76 \mathrm{~g}$ $(58 \%){ }^{1} \mathrm{H} \mathrm{NMR}\left(\mathrm{CDCL}_{3}\right): \delta 7.61 \mathrm{ppm}(\mathrm{s}, 2 \mathrm{H}) ; 4.95 \mathrm{ppm}$ $(\mathrm{s}, 4 \mathrm{H}){ }^{13} \mathrm{C} \mathrm{NMR}\left(\mathrm{CDCL}_{3}\right): \delta 153,131,129,28 \mathrm{ppm}$.

\subsection{Synthesis of 4,7-di(carbaldehyde)-benzo $(1,2,5)$ thiadiazole (2) (DCBT)}

BBT $(0.1 \mathrm{~g}, 0.34 \mathrm{mmol})$ and $\mathrm{NaIO}_{4}(0.03 \mathrm{~g}, 0.11 \mathrm{mmol})$ were dissolved in DMF $(5 \mathrm{~mL})$ and the solution was refluxed at $120^{\circ} \mathrm{C}$ for $24 \mathrm{~h}$ under nitrogen, with the progress of the reaction monitored by thin layer chromatography. The solution was then left to cool and $50 \mathrm{~mL}$ of deionised water added to the solution and the combined solution was extracted with diethyl ether $(2 \times 20 \mathrm{~mL})$. The combined ether fractions were dried with $\mathrm{NaSO}_{4}$ and the ether evaporated, after which compound 2 (DCBT) was obtained by column chromatography (5:1 hexane:ethyl acetate). Yield: $0.052 \mathrm{~g}(78 \%)^{1} \mathrm{H} \mathrm{NMR}\left(\mathrm{CDCL}_{3}\right): \delta 8.3 \mathrm{ppm}$ $(\mathrm{s}, 2 \mathrm{H}) ; 10.9 \mathrm{ppm}(\mathrm{s}, 2 \mathrm{H}),{ }^{13} \mathrm{C} \mathrm{NMR}\left(\mathrm{CDCL}_{3}\right): \delta 190,160$, $132,129 \mathrm{ppm}$.

\subsection{Synthesis of 4,7-(methanyledene-2-(2- thioxothiazolidin-4-one))benzo(1,2,5)thiadiazole(3) (RhBT)}

DCBT $(0.1 \mathrm{~g}, 0.52 \mathrm{mmol})$ and rhodanine $(0.2 \mathrm{~g}, 1.5 \mathrm{mmol})$ was dissolved in $15 \mathrm{~mL}$ of dimethyl sulfoxide and piperidine (3 drops) was added to the solution. The solution was then refluxed and stirred overnight under $\mathrm{N}_{2}$. Once cooled, the solution was added to $20 \mathrm{~mL}$ of water and extracted with dichloromethane. The dichloromethane fractions were collected and evaporated under vacuum, and the remaining viscous liquid was precipitated in water to yield a dark red solid which was purified with column chromatography (DCM followed by ethyl acetate) to yield the product RhBT. Yield: $0.105 \mathrm{~g}(51 \%){ }^{1} \mathrm{H}$ NMR (DMSO-d 6 ): $\delta$ $8.05 \mathrm{ppm}(\mathrm{s}, 2 \mathrm{H}) ; 7.85 \mathrm{ppm}(\mathrm{s}, 2 \mathrm{H}), 4.21 \mathrm{ppm}(\mathrm{s}, 2 \mathrm{H}),{ }^{13} \mathrm{C}$ NMR (DMSO-d $\mathrm{d}_{6}$ ): $\delta 184,173,154,128,127,124 \mathrm{ppm}$. Elemental analysis C: $38.7 \%, \mathrm{H}: 2.3 \%, \mathrm{~N}: 10.4 \%, \mathrm{~S}: 29.7 \%$.

\section{Results and discussion}

\subsection{Synthesis of rhodanine flanked BT}

The molecular acceptor RhBT was synthesised through three steps: bromomethylation of 2,1,3-benzothiadiazole, conversion of the bromomethyl functionality to aldehyde, followed by Knoevenagel condensation [18].

Scheme 1 shows the reaction scheme for the synthesis of RhBT between the dialdehyde and rhodanine. In the first step, bromomethylation of 2,1,3-benzothiadiazole was done using $\mathrm{HBr} /$ Acetic acid and the product (BBT) was obtained in good yields after recrystallization from toluene. The second step is the oxidation of the bromomethyl functionalities into aldehydes, and sodium periodate in DMF was used as the oxidising agent. However, when the reaction was done at high temperatures $\left(150^{\circ} \mathrm{C}\right)$ as reported in the literature, significant amount of 


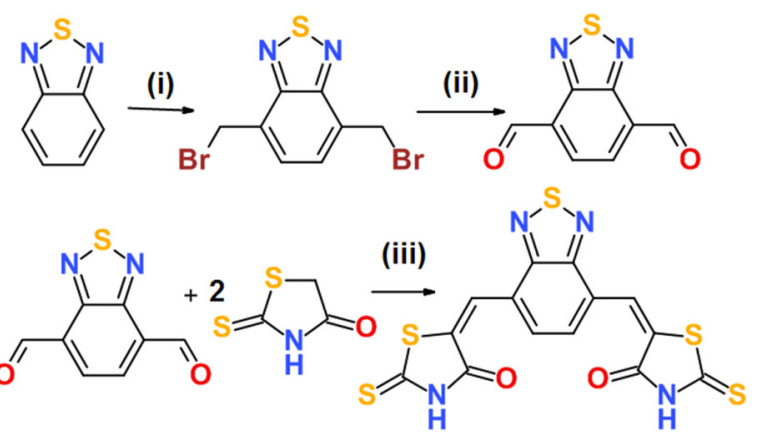

Scheme 1. Reaction scheme for the synthesis of RhBT.

side products were seen as longer reaction times were required to form the di-aldehyde whereas shorter reaction times gave the mono-aldehyde. Similar results were obtained when the amount of oxidising agents was increased, with side products likely containing alcohol functionalities due to further oxidation of the aldehyde. Indeed, longer time and excess oxidant resulted in a product that contains both aldehyde and alcohol functionalities, indicating that one of the aldehyde was further oxidised in the presence of excess sodium periodate. Thus, the ratio of oxidant to starting material was kept low to control the reaction (1:10 molar ratio of bromomethyl groups to $\mathrm{NaIO}_{4}$ ), and the reaction was extended to $24 \mathrm{~h}$ at a lower temperature of $120^{\circ} \mathrm{C}$. The reaction was monitored by thin layer chromatography at regular intervals, where it can be seen that the reaction proceeds firstly towards the monoaldehyde product before the second bromomethyl group is oxidized to produce the dialdehyde. Under these conditions DCBT was obtained as the main product after column chromatography.

RhBT was then synthesised through the Knoevenagel condensation of DCBT and rhodanine in DMSO. Previous studies have used varying solvents including $\mathrm{CHCl}_{3}$ and alcohols, however in our study it was found that due to the low solubility of rhodanine in $\mathrm{CHCl}_{3}$ the reaction did not proceed very readily; while when alcohol $(\mathrm{MeOH})$ was used, the reaction product appeared to precipitate out of the solution, and may contain a mixture of mono- and difunctionalised materials. DMSO was thus chosen as the solvent due to good solubility of the product in the solvent, and the reaction proceeded readily under this condition. After extraction and purification the product was obtained as a dark red solid which was partially soluble in alcohols, but showed the greatest solubility in aprotic polar solvents such as DMSO. The final compound shows a deep orange/ red colour when dissolved in solution, indicating extended conjugation in the material.

\subsection{Optical properties of the RhBT acceptor using UV-Vis spectroscopy}

UV-Vis spectroscopy was done to determine the optical properties of the synthesized RhBT. The absorption spectrum of RhBT in solution (DMSO) and as a film is shown in Figure 1. Both the solution and the film revealed reasonable absorption in the visible and high energy areas, typically ranging $280-700 \mathrm{~nm}$. The spectrum reveals a

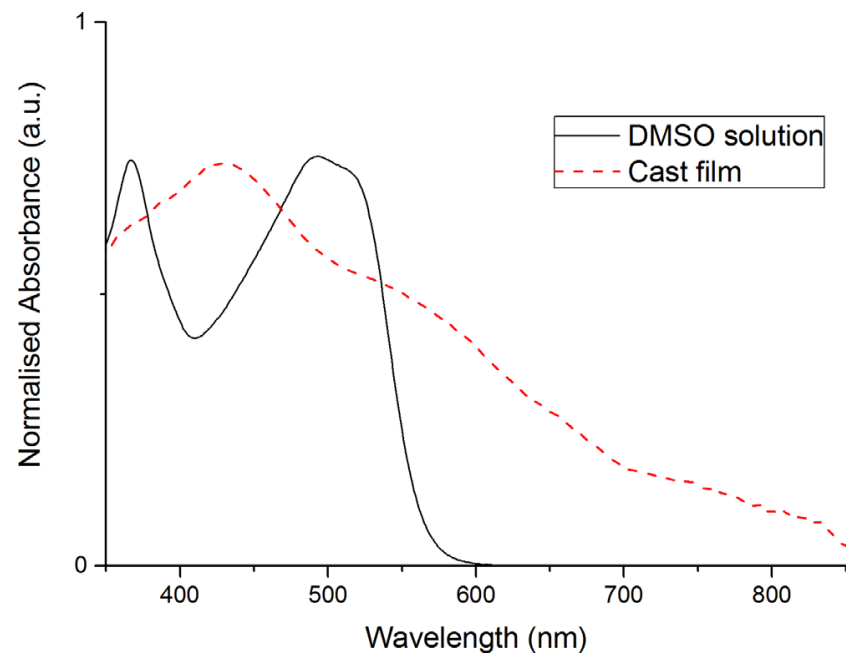

Fig. 1. UV-Vis absorption spectra of RhBT.

maximum absorption of RhBT at $500 \mathrm{~nm}$ in solution and $450 \mathrm{~nm}$ in the thin film. Compared with the maximum absorption of PC60BM (ca. $300 \mathrm{~nm}$ ), RhBT absorbs in a region of the spectrum with much higher solar flux offering significant advantage with regards to contribution to the photocurrent via absorption. The absorption ranging from 280 to $400 \mathrm{~nm}$ also complements well with the absorption characteristics of the donor polymer P3HT [6], which suggests that a blend with this combination of donoracceptor will be able to absorb over an extended absorption range, leading to excellent light-harvesting. In solution, RhBT has an absorption edge at around $600 \mathrm{~nm}$, however when cast as a film and dried the absorption edge tails off further to around $700 \mathrm{~nm}$ although there were noise at higher wavelength due to scattering by aggregates of RhBT in the cast film. This corresponds to a band gap of around $1.7 \mathrm{eV}$. The spectrum also shows two absorption peaks in solution, which appears less well defined in the cast film possibly due to scattering from aggregated particles. These two absorption peaks have been previously observed in benzothiadiazole-containing donor-acceptor type compounds [19], and though the exact source of this remains unknown, it can be attributed to low-lying unoccupied energy levels, strictly localised on the electron-deficient heterocycles yet forming a discrete band of easily accessed energy state within the bandgap of the conjugated system in ground state. Both the solution and the cast film spectra show red-shift when compared with the solution and thinfilm spectra of PC60BM - a conventional fullerene acceptor. Many low energy transitions are hindered in PC60BM due to its high degree of structural symmetry, limiting its absorption in the visible region. This further demonstrates the capacity of this small molecule to yield a greater photocurrent than C60 fullerene acceptors.

\subsection{Electrochemical characterization using cyclic voltammetry}

Cyclic voltammetry (CV) was performed in order to determine the highest occupied molecular orbital (HOMO) and lowest unoccupied molecular orbital (LUMO) levels of 


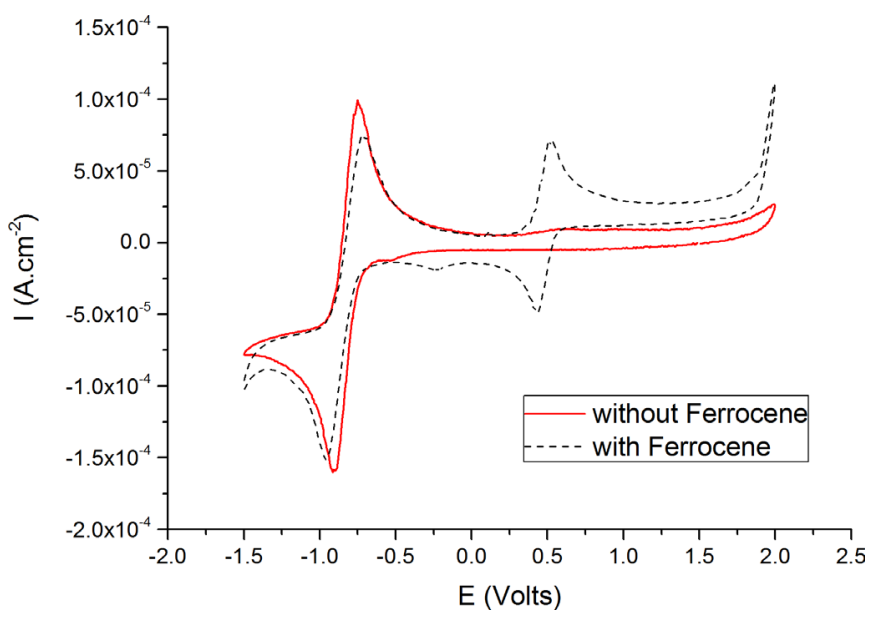

Fig. 2. CV scan of RhBT in acetonitrile with $0.1 \mathrm{M}$ t-butylammoniumhexafluorophosphate as electrolyte and ferrocene internal standard.

the compound, which can be calculated from the empirical relation proposed by de Leeuw et al. [20] of $I_{p}(\mathrm{HOMO})=$ $-\left(E_{\text {onset,ox }}+4.4\right) \mathrm{eV}$ and $I_{p}(\mathrm{LUMO})=-\left(E_{\text {onset,red }}+4.4\right)$ $\mathrm{eV}$ where $E$ and $E_{\text {onset,red }}$ are the onset potentials for oxidation and reduction respectively (vs SCE). CV scan of the compound in Figure 2 shows that RhBT has a reversible reduction peak with an onset of $-0.55 \mathrm{~V}$ vs. NHE (after correction based on ferrocene internal reference). This corresponds to a LUMO level of $-3.85 \mathrm{eV}$. Reversibility of the reduction peak demonstrates that the reduced species is not electrochemically unstable, which is important for the operational stability of devices. There were no clearly defined oxidation peak visible within the range tested (up to $2 \mathrm{~V}$ ), and running the $\mathrm{CV}$ without the ferrocene internal standard did not show a peak overlapping with ferrocene. Thus, it is likely that the oxidation of $\mathbf{R h B T}$ in this case is indicated by the increase in current at higher potentials of around $1.75 \mathrm{~V}$ vs. NHE (after correction based on ferrocene), corresponding to a HOMO energy level of $-6.15 \mathrm{eV}$. These values are within what would be needed for it to function as an acceptor when compared with donor materials such as P3HT, with a LUMO level very similar to that of PCBM $(-3.84 \mathrm{eV})$ [11]. However, the molecule has a nonplanar molecular structure which may facilitate nonanisotropic electron transport, as well as a reduced tendency to crystallize which is likely to prevent large crystalline domains from forming in the bulk heterojunction blend composition over extended lifetimes. The central BT unit offers delocalization potential over the whole molecule, while the lowest unoccupied molecular orbital (LUMO) is concentrated onto the more electron deficient peripheral Rh units. The energy levels indicate the suitability of using these rhodanine acceptors use in conjunction with wide bandgap polymers such as poly(3-hexylthiophene) (P3HT) for OPV application, however, greater aromaticity may bring the HOMO/LUMO levels closer to that of P3HT to increase its efficiency. Extending the conjugation along the compound can be expected to reduce the HOMO level, while the LUMO level can be controlled through the combination of electron donating and withdrawing groups, with previous studies showing that strong electron with- drawing groups resulted in a lower lying LUMO level [16]. Study is currently underway on the effect of substituents on the amino group of rhodanine on its optical and electrochemical properties.

\section{Conclusions}

We have successfully prepared a structurally simple rhodanine-substituted benzothiadiazole compound by a simple method using Knoevenagel condensation of disubstituted benzothiadiazole and rhodanine. The material can be synthesized in high yields via facile synthetic steps. The compound exhibits optical and electrochemical properties suitable for use as molecular acceptor in organic solar cells, with a HOMO and LUMO level of -6.15 and $-3.85 \mathrm{eV}$, respectively. The material offers distinct advantage over fullerene acceptors, for which high cost and lengthy purification have long been a prohibitive factor. Furthermore, RhBT molecule is highly absorbing in the visible region in comparison to fullerene acceptors, offering the potential for enhanced photocurrent generation in devices. In future, donor-acceptor combination study will be undertaken to investigate the effect of different combinations of donors and acceptor materials on solar cell performance.

\section{Author contribution statement}

M.A. and N.R.C. designed the experiments. S.S. performed the experiments and synthesized the acceptor. S.S., N.R.C., M.A., N.K.D. (all in Part) analyzed the data. S.S. wrote the draft with N.R.C., M.A., N.K.D. and the manuscript was finalized through contributions of all authors. All authors have given approval to the final version of the manuscript.

\section{Supplementary Material}

Figure $\mathbf{S 1}{ }^{1} \mathrm{H}$ NMR spectrum of 4,7-di(bromomethyl)benzo $(1,2,5)$ thiadiazole (1)(BBT).

Figure S2 $^{1} \mathrm{H}$ NMR spectrum of 4,7-di(carbaldehyde)benzo(1,2,5)thiadiazole(2)(DCBT).

Figure S3 ${ }^{1} \mathrm{H}$ NMR spectrum of 4,7-(methanyledene-2-(2thioxothiazolidin-4-one))-benzo(1,2,5)thiadiazole(3)

(RhBT).

The Supplementary Material is available at https:// www.epj-pv.org/10.1051/epjpv/2017007/olm.

The authors would like to acknowledge funding and support from the South Australian Government International Research Grant, and Australian Research Council Discovery Grant. Thanks to Dr. Renee Kroon for his contribution in scientific discussions.

\section{References}

1. A.F. Eftaiha, J.-P. Sun, I.G. Hill, G.C. Welch, J. Mater. Chem. A 2, 1201 (2014)

2. W. Chen, Q. Zhang, J. Mater. Chem. C 5, 1275 (2017)

3. J. Zhang, B. Zhao, Y. Mi, H. Liu, Z. Guo, G. Bie, W. Wei, C. Gao, Z. An, Dyes Pigments 140, 261 (2017)

4. J.E. Anthony, Chem. Mat. 23, 583 (2011) 
5. R. Xin, J. Feng, C. Zeng, W. Jiang, L. Zhang, D. Meng, Z. Ren, Z. Wang, S. Yan, ACS Appl. Mater. Interfaces 9, 2739 (2017)

6. D. Srivani, A. Gupta, S.V. Bhosale, Av.L. Puyad, W. Xiang, J. Li, R.A. Evans, S.V. Bhosale, Chem. Commun. 53, 7080 (2017)

7. E. Lim, S. Lee, K.K. Lee, Mol. Cryst. Liq. Cryst. 565, 98 (2012)

8. S. Qu, H. Tian, Chem. Commun. 48, 3039 (2012)

9. Y. Zhou, L. Ding, K. Shi, Y.-Z. Dai, N. Ai, J. Wang, J. Pei, J. Adv. Mater. 24, 957 (2012)

10. P. Hudhomme, EPJ Photovoltaics 4, 40401 (2013)

11. W. Yin, M. Dadmun, ACS Nano 5, 4756 (2011)

12. J. Ray, N. Panja, P.K. Nandi, J.J. Martin, W.E. Jones, J. Mol. Struct. 874, 121 (2008)

13. C.A. Echeverry, A. Insuasty, M.Á. Herranz, A. Ortíz, R. Cotta, V. Dhas, L. Echegoyen, B. Insuasty, N. Martín, Dye Pigment. 107, 9 (2014)
14. Y. Zhou, W. Chen, Z. Du, D. Zhu, D. Ouyang, L. Han, R. Yang, Sci. China Chem. 5, 357 (2015)

15. Q. Zhang, B. Kan, F. Liu, G. Long, X. Wan, X. Chen, Y. Zuo, W. Ni, H. Zhang, M. Li, Z. Hu, F. Huang, Y. Cao, Z. Liang, M. Zhang, T.P. Russell, Y. Chen, Nat. Photonics 9, 35 (2014)

16. S. Holliday, R.S. Ashraf, C.B. Nielsen, M. Kirkus, J.A. Röhr, C.-H. Tan, E. Collado-Fregoso, A.-C. Knall, J.R. Durrant, J. Nelson, I. McCulloch, J. Am. Chem. Soc. 137, 898 (2015)

17. Y. Kim, C.E. Song, S.-J. Moon, E. Lim, Chem. Commun. 50, 8235 (2014)

18. J.U. Ju, S.O. Jung, Q.H. Zhao, Y.H. Kim, J.T. Je, S.K. Kwon, Bull. Korean Chem. Soc. 29, 335 (2008)

19. A. Nowakowska-Oleksy, J. Cabaj, K. Olech, J. Sołoducho, S. Roszak, J. Fluoresc. 21, 1625 (2011)

20. D.M. de Leeuw, M.M.J. Simenon, A.R. Brown, R.E.F. Einerhand, Synth. Met. 87, 53 (1997)

Cite this article as: Surya Subianto, Mats Andersson, Naba Dutta, Namita Roy Choudhury, Novel rhodanine based molecular acceptor for organic solar cells, EPJ Photovoltaics 8, 80402 (2017) 